\title{
An insight on designers' sketching activities in traditional versus digital media
}

Zafer Bilda and Halime Demirkan, Department of Interior Architecture and Environmental Design, Bilkent University, 06533, Bilkent, Ankara, Turkey

This study aims at gaining an insight on designers' cognitive processes while sketching in digital vs traditional media. Empirical data on design processes have been obtained from protocol analyses of six interior designers solving an interior space-planning problem through media transition. In order to encode the design behavior, a coding scheme was utilized that allowed the inspection of both the design activity and the responses to media transition in terms of the primitive cognitive actions of designers. The analyses of the coding scheme constituents, which are segmentation and cognitive action categories, allowed a comparative study demonstrating the effect of the use of different media in the conceptual design phase. The results showed that traditional media had advantages over the digital media, such as supporting the perception of visual-spatial features, and organizational relations of the design, production of alternative solutions and better conception of the design problem. These results also suggested implications for computer aids in architectural design to support the conceptual phase of the design process.

(C) 2002 Elsevier Science Ltd. All rights reserved.

Keywords: design cognition, protocol analysis, sketching, conceptual design, design activity

1 Van Someren, M W, Barnard, Y F and Sandberg, J A C The think aloud method: a practical guide to modelling cognitive processes Academic Press, London (1994)

2 Newell, A and Simon, H A Human Problem Solving Prentice Hall, NJ (1972)

3 Ericsson, $\mathrm{K}$ A and Simon, $\mathrm{H}$ A Protocol Analysis: Verbal reports as data MIT Press, Cambridge, MA (1993)

4 Eastman, C M 'On the analysis of intuitive design processes' in G T Moore (ed.) Emerging Methods in Environmental Design and Planning, MIT Press, Cambridge, MA (1970) pp 21-37

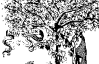

$\mathrm{T}$

The attempts to understand 'how designers design' started in the 1960 s with the method of introspection used by psychologists, where the subject was encouraged to give a coherent verbal report on a cognitive process ${ }^{1}$ then proceeded with innovative methods for analysing the verbal protocols of problem solving ${ }^{2,3}$. The question of how designers think' was not separable from the former question, and many aspects of both questions were explored by using the design protocol ${ }^{4-8}$. The analysis of design protocols formalized the study of the intuitive

www.elsevier.com/locate/destud

0142-694X/03 \$ - see front matter Design Studies 24 (2003) 27-50

PII: S0142-694X(02)00032-7

(C) 2002 Elsevier Science Ltd All rights reserved Printed in Great Britain 
5 Akin, O Psychology of Architectural Design Pion, London (1986)

6 Schon, D A The Reflective Practitioner Temple Smith, London (1987)

7 Goldschmidt, G 'The dialectics of sketching' Creativity Research Journal Vol 4 No 2 (1991) 123-143

8 Goldschmidt, G 'On visual design thinking: the vis kids of architecture' Design Studies Vol 15 (1994) 158-174

9 Akin, $O$ 'How do architects design?' in Unknown Latombe (ed.) Artificial Intelligence and Pattern Recognition in Computer Aided Design, North Holland Publishing Company, IFIP (1978)

10 Do, E, Yi, L, Gross, M D, Neiman, B and Zimring, C 'Intentions in and relations among design drawings' Design Studies Vol 21 No 5 (2000) 483-503

11 Schon, D A and Wiggins, $G$ 'Kinds of seeing and their function in designing' Design Studies Vol 13 (1992) 135-156

12 Goel, V Sketches of Thought MIT Press, Cambridge, MA (1995)

13 Suwa, $M$ and Tversky, B 'What do architects and students perceive in their design sketches? A protocol analysis' Design Studies Vol 18 No 4 (1997) 385-403

14 Suwa, M, Purcell, T and Gero, J S 'Macroscopic analysis of design processes based on a scheme for coding designers' cognitive actions' Design Studies Vol 19 No 4 (1998) 455-483

15 Kavakli, M, Suwa, M, Gero, $\mathbf{J}$ and Purcell, $\mathbf{T}$ 'Sketching interpretation in novice and expert designers' in $\mathbf{J} \mathbf{S}$ Gero and B Tversky (eds) Visual and Spatial Reasoning in Design, Key Center of Design Computing and Cognition, University of Sydney, Sydney (1999) pp 209-219 16 Suwa, M, Gero, J and Purcell, $\mathbf{T}$ 'Unexpected discoveries and S-invention of design requirements: important vehicles for a design process' Design Studies Vol 21 No 6 (2000) 539-567

17 Verstijnen, I M, Hennessey, J M, Leeuwen, C, van Hamel, $\mathbf{R}$ and Goldschmidt, $\mathbf{G}$ 'Sketching and creative discovery' Design Studies Vol 19 No 4 (1998) 519-546 aspects of design and has been the basis of design cognition studies, revealing important insights into design problem solving and sketching in the architectural context.

Sketches, being an integral part of design, store the design solutions and also seem to be essential for recognizing conflicts and possibilities ${ }^{9}$ as well as for revising and refining ideas, generating concepts and facilitating problem solving ${ }^{10}$. Therefore, design research has examined extensively the early stages (conceptual phase) of the design process and the role of sketching. Schon ${ }^{6}$ described the design activity as a reflection-in-action process, Goldschmidt ${ }^{7}$ introduced 'seeing as' and 'seeing that' notions to explain design reasoning, then free-hand sketches in design activity has been emphasized to be an essential medium in designers' dialectic process $^{7,11,12}$. Based on Schon and Wiggins' work $^{11}$, Suwa and Tversky ${ }^{13}$ examined what information architects think of and read off from their own freehand sketches. Also Suwa et al. ${ }^{14}$ explored sketches to find how they can be a good medium for a reflective conversation. The serial studies of design protocols revealed insights on different aspects of the design activity $^{15,16}$.

Most of these studies based on design protocols have used free-hand sketches as a medium to analyze the design activity. Also, digital sketching as a medium can be studied, as there is little research on comparing the traditional versus digital media using design protocols. The reason is that hand-sketches have mostly been regarded as the medium of thought processes, and digital sketches are claimed not to be supporting creativity in the conceptual design phase ${ }^{17,18}$. On the contrary, Madrazo ${ }^{19}$ claimed that digital visual representations can be used for better understanding of the form, thus as a support for visual thinking. Marx ${ }^{20}$ supported this idea by pointing out that intensive visualization and immediate feedback in computer media influenced the designer to generate imaging in his/her mind more frequently, compared to conventional media ${ }^{21}$. This gives evidence that designer's visual thinking could be different in digital media. as inferred by Bilda et al. ${ }^{22}$. Yet, the cognitive processes of designers in both media should be analyzed, to explore the effects of media transition and different media interactions of designers. This could establish a framework to propose ways to integrate computers as a medium into conceptual design. Generation of new computer-aided architectural design (CAAD) tools based on human designing behaviors could possibly be achieved by using the results of design cognition research for $\mathrm{CAAD}^{23}$.

The current study employs a task analysis in terms of goals and subgoals of designers and introduces a content-oriented coding method based on the 
18 Elsas van, P A and Vergeest, J S M 'New functionality for computer aided conceptual design: the displacement feature' Design Studies Vol 19 No 1 (1998) 81-102

19 Madrazo, L 'Types and instances: A paradigm for teaching design with computers' Design Studies Vol 20 (1999) 177-193

20 Marx, J 'A proposal for alternative methods for teaching digital design' Automation in Construction Vol 9 (2000) 19-35

21 Won, P H 'The comparison between visual thinking using computer and conventional media in the concept generation stages of design' Automation in Construction Vol 10 (2001) 319-325

22 Bilda, Z, Demirkan, $H$, and Erkip $\mathrm{F}$ Traditional versus digital design medium: A case study in the interior design studio Unpublished manuscript, Bilkent University, Bilkent, Ankara, Turkey (2000) available on the World Wide Web: www.bilkent.edu.tr/ zafer

23 Tang, H-Hui, and Gero JS Content-oriented coding scheme for protocol analysis and computer-aided architectural design (2000) Retrieved 2001, from the World Wide Web: www.arch. usyd.edu .au/ john/publications 24 Simon, H A 'The structure of ill-structured problems' Artificial Intelligence Vol 4 (1973) 181-201

25 Doorst, $\mathrm{K}$ and Dijkuhuis, J 'Comparing paradigms for describing design activity' Design Studies Vol 16 No 2 (1995) 261-274 cognitive characteristics of designers-adapted from the coding scheme proposed by Suwa et al. ${ }^{14}$, in order to inspect the design behaviour in traditional vs digital media. The task analysis together with encoded cognitive activities enabled us to get an insight on designers' reasoning steps, interrelations among them and on the problem solving strategies. The change of media in the experiment enabled us to compare the problem solving behavior in different media and the effect of learning. As a result the paper envisions general implications on problem solving behaviors and sketching activities of the designers in paper-based media vs the CAD environment.

\section{The protocol analysis method}

Problem solving could be characterized as a cognitive process that is goal directed, in which the solution emerges via intermediate reasoning steps some of which may later appear useless or false ${ }^{1}$. Analysis of these reasoning steps would underlie the differences and commonalities of subjects' thinking, learning and problem solving behaviors. Simon ${ }^{24}$ defined design activity as an ill-structured problem solving process where the problem and its solution are both not well defined. For dealing with the ill-structure and nature of design as problem solving activity, Simon ${ }^{24}$ proposed decomposing the problem into goals and subgoals where the designer finds partial solutions for subgoals. Further, he proposed the examining of interrelations between subgoals to understand the decision- making process of designers. This type of procedure was defined as the process-oriented approach by Dorst and Dijkhuis ${ }^{25}$ and the other, which deals with 'what designers see, attend to, think of and retrieve from memory while designing ${ }^{14}$ was named as the content-oriented approach (p. 457). There are several methods of inspecting the process of problem solving, like observation of behavior, observation of structured dialogues, and analysis of verbal reporting (protocols), which could be concurrent or retrospective ${ }^{1}$. Starting with Suwa and Tversky ${ }^{13}$, recent protocol analysis methods have used restrospective reporting ${ }^{14,15,16}$ that employs both segmentation and encoded categories of cognitive actions. The protocol analysis method used in the present study also employs similar retrospective reporting, a segmentation scheme and a coding scheme (basically like Suwa et al.'s scheme ${ }^{14}$ ). Based on the interior space planning process we have devised a segmentation scheme considering that a subject solves a problem in terms of a hierarchy of subgoals. Similarly, Newell and Simon ${ }^{2}$ introduced 'problem solving episodes' to analyze the protocols at an aggregate level where the episodes were defined as 'aspects of the problem that can be viewed as demands of the task environment' (p. 283). So a task analysis was done on the problem space by observing the action protocols of designers, which formed some "obvious" categories that we used to segment the raw protocols. 
Then for each segment, the cognitive actions of designers are coded into four action categories. The coding scheme was based on a psychological model where the four categories of the coding scheme corresponded to the levels at which incoming information was thought to be processed in human cognition ${ }^{12}$. Thus the present study employs an analysis procedure with a content-oriented coding scheme and a process-oriented method of segmentation, which seem more appropriate for an interior space-planning problem solving process.

\subsection{Segmentation categories}

The interior space planning process is like a trial and error process that the designer chooses among alternatives of size, hierarchy, and organization of spaces as well as organization of spatial elements regarding some human, technical and psychological factors and other specific requirements of the clients. Those factors converge the design problem to a goal directed process, so it is reasonable to divide the design process into units according to the goals that designers most attend in common. After each design session and protocol, a simple model of the design process in each session is documented by noting down the goals achieved. The drafts of the documented data help to form five categories based on the most common goals and intentions and one defined state for each media (Table 1). Category A was creating/defining a space component that includes either of the acts that are mentioning of a space by a pencil stroke, clearly drawing the space component, defining the invisible boundaries of a space by only putting things/objects into that intentional space, or the combination of those previous intentions. Category B was defined as the revisit of a space component for several purposes such as adding space elements, furniture, equipment, or associating relations among them. Category $\mathrm{C}$ was assessed to be the redefinition of a space component, which might occur either by geometrical transformation of a space or by changing the organization of the elements/things/objects that constitute or bind the space so that the space is redefined. Category D included the association of the local and global relations in the design problem, while Category $\mathrm{E}$ included the redefinition of those global or local relations. We have also assessed two states of the design activity in the segmentation categories, which were 'reproduction of the design' ( $F$ type segment) specific to the traditional media and 'looking at the 3D view' (G type segment) specific to the digital media. 'Reproduction of design' was present only in the hand-sketch media, which involved the copying of design on a new sheet of tracing paper. Similarly the $\mathrm{G}$ state (looking at the 3D view) occurred only in CAD sketches where the $3 \mathrm{D}$ (camera) view was available. The $\mathrm{G}$ state is accepted as a segment if the instant intention of the designer was to check spatial relations or functional issues. However the G state is also applicable in the body of 
A. Define/Create a space component or an area

1. Mentioning the space only (gesture, circle, rectangle, line) or draw the space component or area.

2. Put things/objects into the space component.

Drawing spatial elements (doors, windows, etc.)

Drawing furniture (table, sofa, accessories, etc.)

Drawing equipment (handbasin, bathtub, etc.)

3. Combination of $1 \& 2$.

4. Substituting a space component with another one that had a different function.

B. Revisit the space component for details

1. Adding space elements (window, door, etc.) or/and furniture/accessories, equipment.

2. Associate local relations among objects/things (organization).

3. Combination of $2 \& 3$.

C. Redefine the space component

1. Transformation of a space component (scale/translate/rotate/change the geometry/combination)

2. Change the organization of furniture/equipment

According to geometry principles (adjacency/symmetry/reflection)

According to functional requirements.

Combination

3. Combination of $1 \& 2$.

D. Definition of spatial relations

1. Associating global relations (private/semiprivate/public areas)

2. Associating local relations between space components (putting door, opening, wall, A1 \& A2)—the design intention is extracted from the protocol.

E. Redefinition of (a) spatial relation(s)

1. Redefinition of global relations

2. Redefinition of local relations

By changing the location of a space component

By changing the location of doors or reorganizing things/objects in or between the space components.

F. Reproduction of design

Copy the design/tracing on a new sheet/redraw the layout

G. Looking at the $3 D$ view (digital media)

This action becomes a segment when there is a focus shift. It is not defined as a segment when the action takes place to check the design actions/decisions in the same segment.

other types of segments for occasions when the designer checks the attributes of the space elements.

The segmentation in this study employed segments that reflected the designer's intentions in terms of their goals and subgoals in the specific design problem. Each designers' session had different numbers of segments, depending on variables like the designer's way of problem solving, procedure of decision-making, learning effect, the media and so on. It is not claimed that these categories cover all the possible goals and actions of the designer in the interior space planning problem-solving process, but it was possible to decompose all the design sessions in the experiment in terms of those categories. The categories were classified by analyzing the verbal protocols and watching the videotapes of a number of times. When the designer changes his/her strategy or intention this should flag the start 
of a new segment. Sometimes this change might not occur clearly, but with the visual aid of the videotaped process, the designer remembers his/her intention most of the time. As long as the change in intention is clear in the verbal protocol, both the start-end and the type of the segment could be marked.

\subsection{Action categories}

Each segment is comprised of various kinds of cognitive actions. These actions were classified into four categories, namely as physical, perceptual, functional and conceptual by Suwa et al. ${ }^{14}$. These four categories corresponded to the levels at which incoming information was thought to be processed in human cognition ${ }^{14}$. The present study used the same action categories, but the encoded cognitive actions were revised, in order to provide an insight to the comparison of design activity in traditional vs digital media (Table 2).

The four major encoded categories included subcategories. 'Physical actions' are divided into three subcategories named, 'draw', 'modify' and 'copy'. The 'perceptual action' category is divided into three, which are named as 'features', 'relations' and 'implicit' subcategories. As described in Suwa et al. ${ }^{14}$ 'features' refer to visual and spatial attributes of depicted elements such as their shape, size or texture. The 'relations' subcategory defined spatial and organizational relations among elements, objects/things such as connectedness, alignment, and remoteness. The last one was related to 'implicit' spaces, which included a single action of discovering a space as ground. The 'functional' category was divided into three subcategories named, 'implement', 'reinterpretation' and 'thought' functions. 'Implement' refers to the functions related to the implementation of functional criteria that the designer makes up in his/her strategies. 'Reinterpretation' of a function has only one encoded cognitive action. The last subcategory 'thought functions' was related to the functions that the designer thinks of during the design process. Finally 'the conceptual actions' category had 'setting up of goals' and 'retrieval of knowledge' actions. The details of the action categories can be seen in Table 2 .

\section{The experiment}

The subject group consisted of six graduate students (of whom three were male and three female) of the Interior Architecture and Environmental Design Department at Bilkent University. The six participants were voluntary and had acquired the same amount of knowledge and experience in CAD. They had completed the two undergraduate AutoCAD courses, one dealing with 2-dimensional (2D) drafting and the other with the 3-dimensional (3D) modeling. 


\begin{tabular}{|c|c|c|c|}
\hline Action & Subcategory & Action ID & Description \\
\hline \multirow[t]{6}{*}{ PHYSICAL } & \multirow[t]{2}{*}{ Draw } & $\mathrm{Dc}$ & $\begin{array}{l}\text { Making new depictions (drawing lines, } \\
\text { walls, things which are object, furniture, } \\
\text { accessories, space elements etc.) }\end{array}$ \\
\hline & & Dsy & $\begin{array}{l}\text { Depicting a symbol that represents a } \\
\text { relation (for hand-sketch only) }\end{array}$ \\
\hline & \multirow[t]{3}{*}{ Modify } & Drf & $\begin{array}{l}\text { Revising the shape, size, or texture of a } \\
\text { depiction. These refer to stretching lines } \\
\text { or areas, editing shape, color or texture } \\
\text { in the CAD environment. }\end{array}$ \\
\hline & & $\mathrm{Dd}$ & $\begin{array}{l}\text { Erasing a depiction/delete a wall or } \\
\text { object. }\end{array}$ \\
\hline & & Md & $\begin{array}{l}\text { Moving a depiction/object. Rotate an } \\
\text { object. }\end{array}$ \\
\hline & Copy & Dts & $\begin{array}{l}\text { Tracing over a depiction on a new sheet } \\
\text { of paper (for hand-sketch only) }\end{array}$ \\
\hline \multirow[t]{7}{*}{ PERCEPTUAL } & \multirow[t]{3}{*}{ Features } & Pfn & $\begin{array}{l}\text { Attending to the feature of a new } \\
\text { depiction (shape, angle, size, texture) }\end{array}$ \\
\hline & & Pfnp & $\begin{array}{l}\text { Attending to the feature of a view in } 3 \mathrm{D} \\
\text { (imagery or graphical) }\end{array}$ \\
\hline & & $\mathrm{Pv}$ & $\begin{array}{l}\text { Creating or attending to a spatial relation } \\
\text { between two space components or area } \\
\text { (symmetrical, adjacent, far, on the same } \\
\text { axis...) }\end{array}$ \\
\hline & \multirow[t]{3}{*}{ Relations } & Prn & $\begin{array}{l}\text { Creating or attending to a relation } \\
\text { between two objects/things }\end{array}$ \\
\hline & & Plo & $\begin{array}{l}\text { Attending to the location of an object in } \\
\text { a space component (alignment or } \\
\text { geometrical definition) }\end{array}$ \\
\hline & & Prp & $\begin{array}{l}\text { Discovering an organizational relation } \\
\text { between things/objects (more than two } \\
\text { things/objects) }\end{array}$ \\
\hline & Implicit & Psg & Discovering a space as ground \\
\hline \multirow[t]{5}{*}{ FUNCTIONAL } & \multirow[t]{2}{*}{ Implement } & Fn & $\begin{array}{l}\text { Associating a new depiction, feature or } \\
\text { relation with a specific function that was } \\
\text { previously thought or newly discovered }\end{array}$ \\
\hline & & $\mathrm{Fi}$ & $\begin{array}{l}\text { Implementing a previously explored or } \\
\text { through function be creating a new } \\
\text { depiction, feature or relation }\end{array}$ \\
\hline & Reinterpretation & Fre-I & Re-interpretation of a function \\
\hline & \multirow[t]{2}{*}{ Thought functions } & $\mathrm{Fc}$ & $\begin{array}{l}\text { Thinking of a function to be seen } \\
\text { implemented. }\end{array}$ \\
\hline & & Fnp & $\begin{array}{l}\text { Thinking of a function independently of } \\
\text { depictions }\end{array}$ \\
\hline \multirow[t]{2}{*}{ CONCEPTUAL } & Goal setting & G & $\begin{array}{l}\text { Setting up of goals. Introducing new } \\
\text { functions, resolving problematic conflicts, } \\
\text { and goals to apply introduced functions } \\
\text { or arrangements [14] }\end{array}$ \\
\hline & \multicolumn{2}{|l|}{ Knowledge retrieval } & Retrieve knowledge. \\
\hline
\end{tabular}


The experiment was conducted in two settings, an office and a CADlab. In the CADlab, a second monitor and a video recorder were connected to the PC to record the digital design process. In the office, a camera was placed above the sketching desk which was connected to a TV (far from the designer) and a video recorder to monitor and videotape the design process.

The Design Apprentice (www.designapprentice.com) software used in the experiment is an easy to use commercial software, developed especially for interior space planning tasks. While using the software, the design proceeds with space elements such as wall, window, door, column and furniture, instead of lines. The software has many objects in its library so that the designer can revise the shape, size and texture of them to fit into his/her design. While drawing the plan, the user has the opportunity to switch to a $3 \mathrm{D}$ view (isometric or camera view) to inspect the changes interactively in the environment.

\subsection{First phase}

The first phase of the experiment consisted of a two-week training programme aiming to provide all subjects with equal proficiency in using the Design Apprentice software. The participants were experienced in designing a given layout on screen with no time limit. After finishing the sketch problem, each participant was asked to explain his/her design decisions and preferences in a dialogue. This was not only a rough rehearsal of retrospective reporting, but also a way of enquiry on participants' profiles.

\subsection{Second phase}

The second phase consisted of three consecutive design sessions. The six participants were divided into two groups randomly. For a simplistic representation in the paper the traditional media will be referred to as HAND and the digital media will be referred to as CAD. Group 1 (one female and two male participants) had the design sessions in CAD-HAND-CAD media repectively and Group 2 (one male and two female participants) had the design sessions in HAND-CAD-HAND order. In the first design session, the designer was required to solve the design problem 1 , in the second sesssion design problem 2 and finally in the third session problem 1 again. The reason for giving problem 1 again is to test the learning effect and to control the changing of variables in the design process.

The participants were given $1 \mathrm{~h}$ for each design session and a 10 min break in between. The design brief (Fig. 1) consisted of a problem definition for an interior space planning, with a given layout and a specification sheet stating the client's requirements. The participants were required to design a flat for four friends with given space components. The most important 


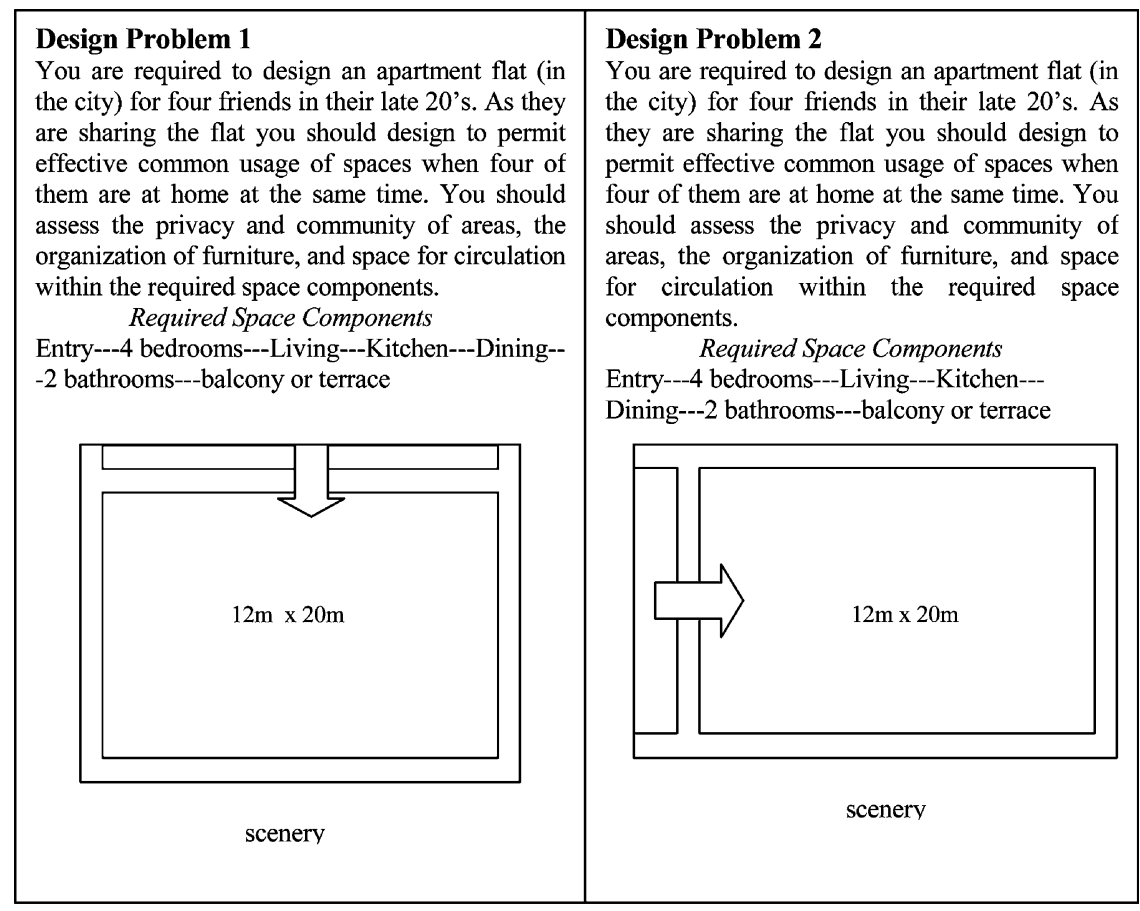

Design Specification Sheet

1. Each component have to be in the following areas

\begin{tabular}{lll}
\hline Public area & Semi-private area & Private area \\
\hline Entry & Kitchen & Bedrooms (4) \\
& Living & Bathrooms \\
& Dining & \\
\hline
\end{tabular}

2. The entry should have easy access to kitchen, dining, living.

3. The kitchen should be open to dining and living space.

4. Satisfy the circulation in the kitchen.

5. Living should be adjacent to the outdoor component, and should face the scenery.

6. Use glass doors opening to outdoor component in appropriate size and location for daylight and to view the scenery in living area.

7. The living space should have two separate spaces, TV lounge and chat lounge.

8. The boundaries of the two separate spaces in living area should be defined by the organization of the furniture.

9. The living space should have furniture for at least four people in each lounge.

10. Two bedrooms should have direct access to one bathroom.

11. Similar size for all bedrooms (might be in different geometry).

12. Sufficient space for bed and study unit in bedrooms.

13. Use high windows at one wall of bedrooms.

Figure 1 Design brief

Designers' sketching activities 
feature required in the design problem was the interior space planning with functions achieved through appropriate room sizes, internal relationships and spatial hierarchy. The specification sheet (Fig. 1) states these requirements in detail. The given boundary was $12 \mathrm{~m} \times 20 \mathrm{~m}$ rectangle and the location of the scenery was indicated. The problem definition and the specifications were the same in two design problems, but the only difference is at the configuration of entry in the layout. The participants were not asked to report concurrently what was going on in their minds, so they were not interrupted by the experimenter. Instead the three design sessions were videotaped.

\subsection{Third phase}

Following the design task, the participants were asked to remember and report what they were thinking while drawing each portion of the sketch. They watched their videotaped sessions while reporting, so that they could recall the design thoughts by the visual aid. Table 3 shows an excerpt from the protocols of a subject employing a CAD-sketch session in Group 2.

Table 4 shows the encoded categories of the above protocol segments.

Table 3 An example of a verbal protocolab

\section{Segment Protocols}

The bathrooms are too big. So, I decided to change the bathrooms. And I make the size smaller (v: stretching the wall), to the rooms' size. I see that corridors emerged. I try to locate the bathtub...I chose a closet and located it. Then I select a door type, and plug it in the walls (v: of two bathrooms). I liked the place of doors. I placed the doors near to rooms, not near to each other. Ok, one more thing, I put the closets adjacent to each other in the bathrooms, and also the bathtubs, they all share the same wall, I thought on ease of plumbing. Now I think on this place (p: emerged corridor space). There is a loss of space here, and I want to add this space to semi-private area. I thought that could be the place for kitchen. Is is good that wet spaces would be together, but then I realized that the space would be narrow and will be in between chat and TV lounges. Further, I couldn't solve the problem there, so I changed my mind then. I found a new solution, later.

20 Now I'm looking for a wardrobe and types of beds, and drawers. I chose a wardrobe and put two wardrobes to here (p: two bedrooms) and here. Then I did the same thing for the others. Now the two rooms at the corners are symmetric, adjacent ones are symmetric, and bathrooms are symmetric also. When I look from here (p: living area) to there (p: private area), I can't see the doors of the bedrooms; they are hidden, that's what I want. And when I'm here (p: in front of the lobby), I only see that wall, and doors of bathroom and bedroom are hidden, and we can hang a picture on that wall by lighting it in a theatrical effect. I search for furniture to put in the bedroom. I put a desk, near window. I select a bed type but do not locate it. I move the desk, and then place the bed there. I choose a drawer. I place the other beds, and then drawers next to them. I zoom in to bedrooms, to place the desks precisely, and chairs, which I've selected. Then I rotate the chairs a little.

\footnotetext{
${ }^{\mathrm{a}}$ (p...) - pointing to areas or things when reporting

b (v:...) - the visual cue in the video recording
} 


\begin{tabular}{|c|c|c|c|c|c|}
\hline $\begin{array}{l}\text { Segment } 18 \\
\text { type C3 }\end{array}$ & & $\begin{array}{l}\text { Segment } 19 \\
\text { type A3 }\end{array}$ & & $\begin{array}{l}\text { Segment } 20 \\
\text { type B3 }\end{array}$ & \\
\hline Physical & & Physical & & Physical & \\
\hline ID & Content & ID & Content & ID & Content \\
\hline Dc 1 & Bathtubs (2) & Drf & Wall & Dc 1 & Wardrobe (4) \\
\hline Dc 2 & Closets (2) & & & Dc 2 & Desk (2) \\
\hline \multirow[t]{2}{*}{ Dc 3} & Bathroom doors & & & Dc 3 & Bed (2) \\
\hline & $(2)$ & & & Dc 4 & Drawer (2) \\
\hline Dc 4 & Sinks (2) & & & Dc 5 & Chair (2) \\
\hline \multirow[t]{2}{*}{ Drf } & $\begin{array}{l}\text { Stretch the } \\
\text { bathroom wall }\end{array}$ & & & Md 2 & $\begin{array}{l}\text { Rotate the chairs } \\
\text { (2) }\end{array}$ \\
\hline & & & & Md 1 & Move the desk \\
\hline Perceptual & & Perceptual & & Perceptual & \\
\hline ID & Content & ID & Content & ID & Content \\
\hline \multirow[t]{2}{*}{ Plo 1} & Locate the & Pfn & Space would be & Plo & Desk \\
\hline & bathroom doors & & narrow & Prn & Relation btw \\
\hline \multirow[t]{2}{*}{ Plo 2} & Locate the & Pfnp & In between & & window and desk \\
\hline & bathtub & & lounges & Py 1 & Doors are hidden \\
\hline \multirow[t]{2}{*}{ Psg } & $\begin{array}{l}\text { Discover a } \\
\text { corridor space }\end{array}$ & & & Pv 2 & $\begin{array}{l}\text { Picture on the } \\
\text { wall with } \\
\text { lighting }\end{array}$ \\
\hline & & & & Pfnp & $\begin{array}{l}\text { Room } \\
\text { organizations } \\
\text { symmetric }\end{array}$ \\
\hline Functional & & Functional & & Functional & \\
\hline ID & Content & ID & Content & ID & Content \\
\hline Fn 1 & $\begin{array}{l}\text { Bathroom doors } \\
\text { are near to } \\
\text { rooms }\end{array}$ & Fnp & $\begin{array}{l}\text { Wet spaces } \\
\text { together }\end{array}$ & $\mathrm{Fh}$ & $\begin{array}{l}\text { Privacy of the p- } \\
\text { area satisfied } \\
\text { (doors are }\end{array}$ \\
\hline Fn 2 & $\begin{array}{l}\text { Bathtubs and } \\
\text { closets share the } \\
\text { same wall }\end{array}$ & & & & hidden) \\
\hline Conceptual & & Conceptual & & Conceptual & \\
\hline ID & Content & ID & Content & ID & Content \\
\hline \multirow[t]{2}{*}{ G 1} & $\begin{array}{l}\text { I liked the place } \\
\text { of doors }\end{array}$ & G 1 & $\begin{array}{l}\text { That space could } \\
\text { be kitchen }\end{array}$ & G 1 & $\begin{array}{l}\text { Hang a picture } \\
\text { on the wall }\end{array}$ \\
\hline & & & & G 2 & $\begin{array}{l}\text { Place the desks } \\
\text { precisely }\end{array}$ \\
\hline
\end{tabular}

\section{Results}

Results are presented in two sections that constitute the analysis of data related to segmentation categories and cognitive actions. For simplicity, cognitive actions will be referred to as $\mathrm{CA}$ in the study. The numbers next to the name of the media represents the session number, so HAND 1 denotes the case where the first problem session was conducted in traditional media. Another common term is 'frequency' which refers to the occurrence of a particular action in percentiles throughout the entire design session. 


\subsection{Analysis of data related to segmentation categories}

It was tested if the segmentation categories were independent of subject performances in design sessions of two media. A Chi-Square Test $\left(\chi^{2}\right)$ was conducted by using the mean number of segmentation categories in all CAD or HAND sessions vs media performances of subjects. There is no evidence $\left(\chi^{2}=3.09, d f=10, p>0.95\right.$ for CAD media; $\chi^{2}=5.163, d f=10$, $p>0.95$, for HAND media) that the segmentation category scheme is related to the subjects' performances in the two media.

The total number of segments in each session of each individual designer is shown in Fig. 2. Subjects' behavior having the HAND-CAD-HAND sessions had a common decreasing pattern (Fig. 2b).

\subsection{Analysis of data related to cognitive actions (CA)}

A $\chi^{2}$ test was done to test if action categories were independent of the media $\left(\chi^{2}=0.96 ; p>0.95 ; d f=3\right)$. There is no evidence that the action

(a)

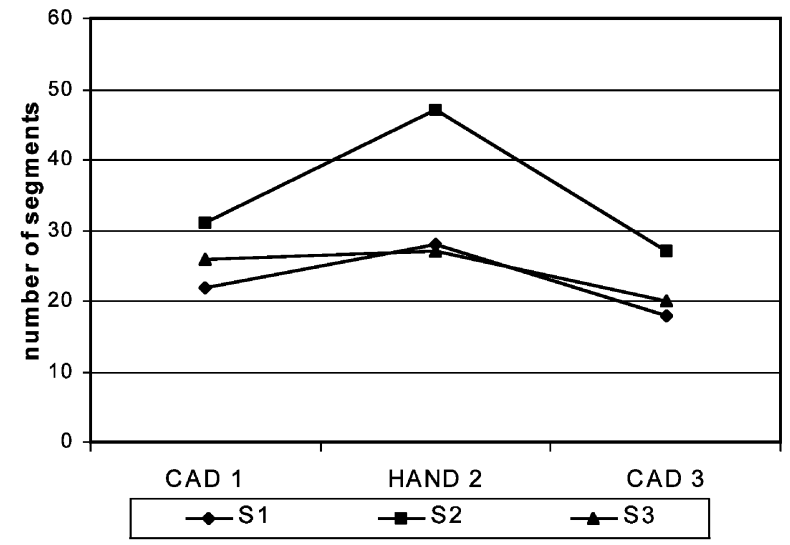

(b)

Figure 2 Total number of segments in (a) CADHAND-CAD sessions (b) HAND-CAD-HAND sessions

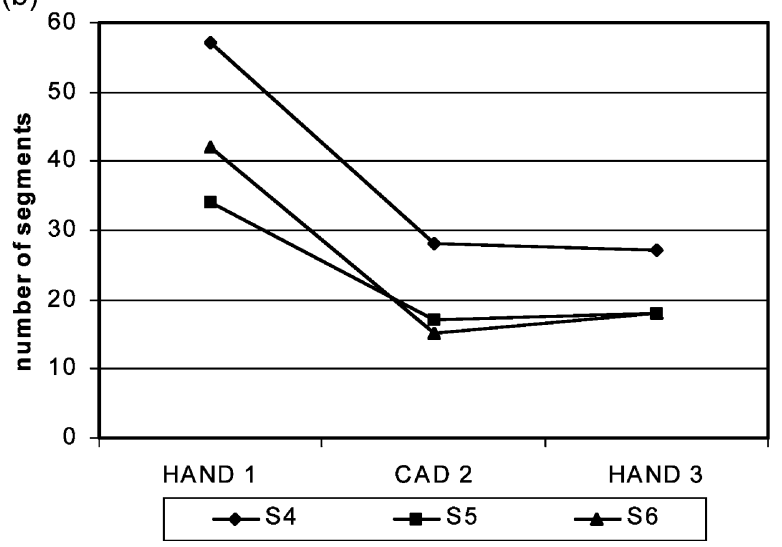


categories are related to the introduced media. The total numbers of CA in consecutive sessions of CAD-HAND-CAD and HAND-CAD-HAND for each individual designer are shown in Fig. 3. It is seen that more cognitive actions are involved in hand-sketch media (HAND), independent of the sequence of the media introduced.

\subsubsection{Physical actions}

As seen in Fig. 4, the frequency of the physical actions in CAD 1 sessions was higher than HAND 1 sessions.

It was observed that there was a major difference between the draw and modify actions in CAD vs HAND media. The mean frequency of 'draw' actions in all CAD sessions (72\%) were lower than all HAND sessions (87\%). 'Modify' actions are more frequently used in CAD environment (28\%) when compared to the traditional media (13\%) and the occurrence

(a)

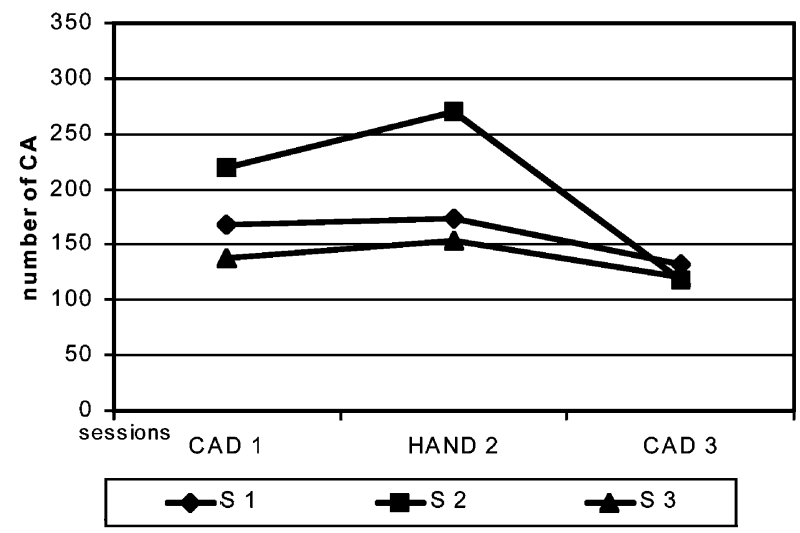

(b)

Figure 3 Total number of $C A$ in (a) CAD-HAND-CAD sessions (b) HAND-CADHAND sessions

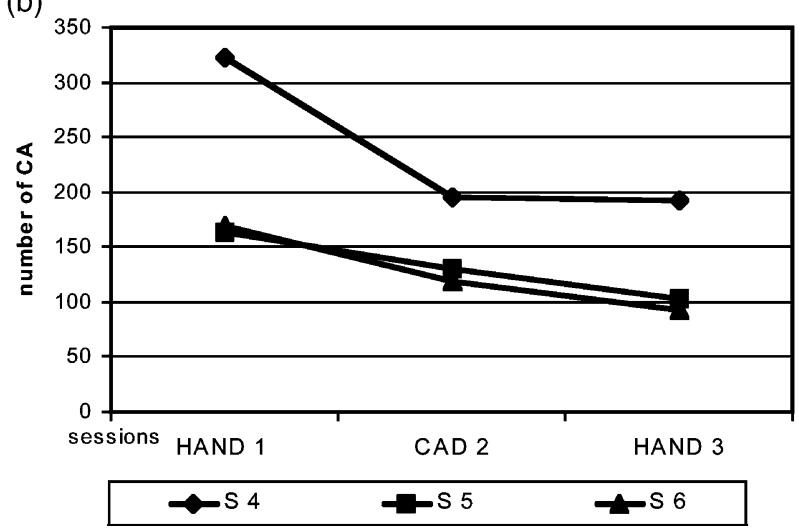


Figure 4 Frequency of physical actions (a) CADHAND-CAD sessions (b) HAND-CAD-HAND sessions

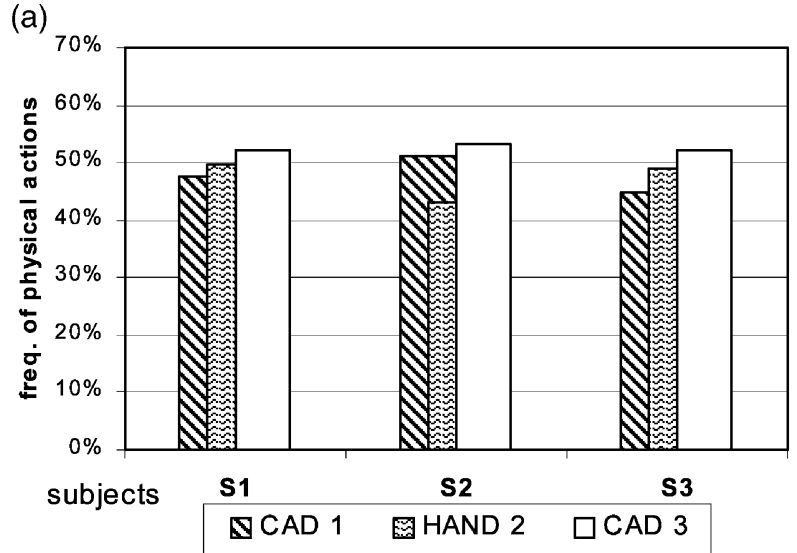

(b)

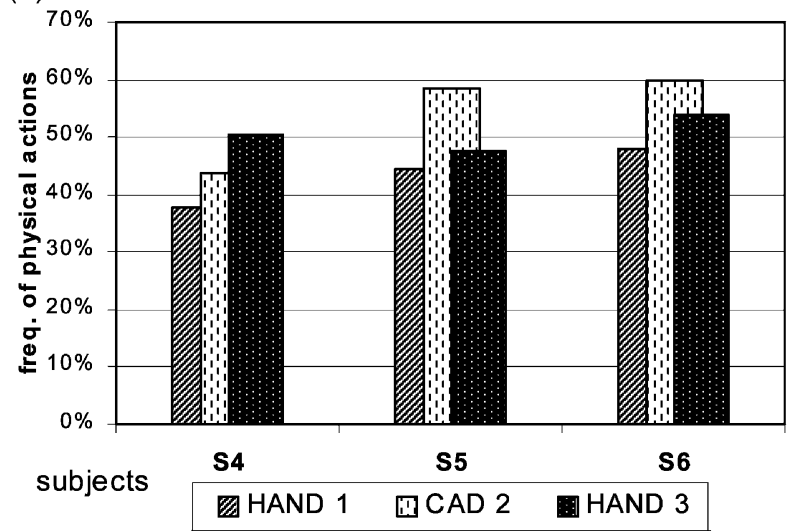

of modification actions was higher in CAD media being independent of the consecutive problem sessions.

\subsubsection{Perceptual actions}

The frequency of the perceptual actions in all sessions seems to be higher in hand sketches as depicted in Fig. 5. When there is a change from HAND 1 to CAD 2 there is a decrease in frequencies of perceptual actions, and when there is a change from CAD 1 to HAND 2 there is an increase (except subject 1) in the frequencies of perceptual actions. This supports the claim that the frequency of perceptual actions is higher in traditional design medium.

The normalized values are used in Fig. 6, in order to inspect the changes in the perceptual actions subcategories, namely 'features' and ' relations' (see Table 2). The normalization process was used to understand where the actual value stands, above or below the average of the action's fre- 
(a)

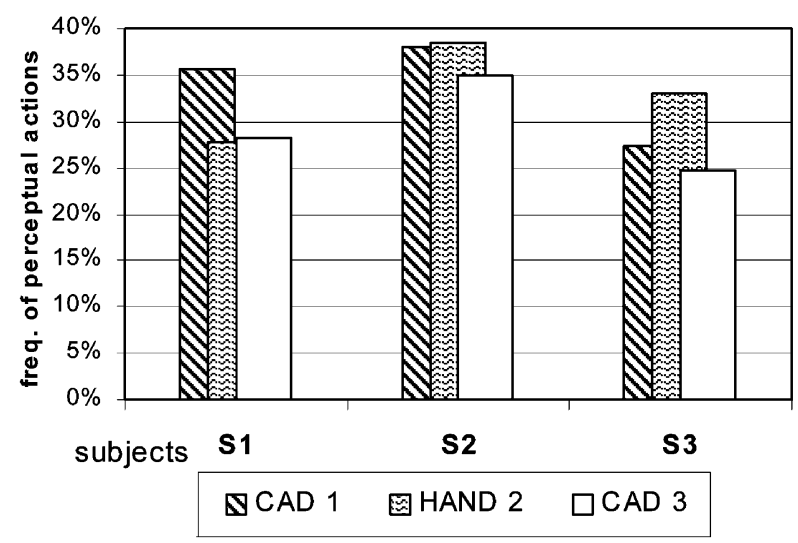

(b)

Figure 5 Frequency of perceptual actions (a) CADHAND-CAD sessions (b) HAND-CAD-HAND sessions

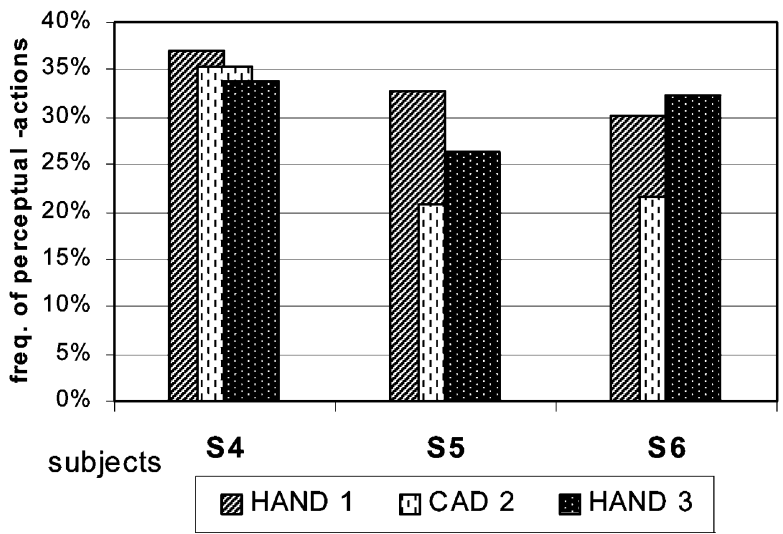

quencies in the 18 sessions. The normalization equation is $\left(\mathrm{F}_{X}-\right.$ $\left.\mathrm{F}_{\text {Xavg }}\right) / \mathrm{F}_{\text {Xavg }}$, where $\mathrm{F}_{X}$ is the actual frequency of a particular action $\mathrm{X}$ and $\mathrm{F}_{\text {Xavg }}$ is average frequency throughout the 18 sessions.

The cognitive actions in the 'relations' subcategory tend to increase (except subject 1) throughout the three consecutive design sessions independent of the media (Fig. 6(a)). On the contrary, the perceptual actions related to the visual-spatial features of the depictions (see Fig. 6(b)) tend to decrease (except subject 1) throughout the three consecutive design sessions.

\subsubsection{Functional-conceptual actions}

As previously stated, both functional and conceptual action categories belong to the semantic level where the information was claimed to be processed $^{14}$. In order to have an insight on the semantic level as a whole, the frequencies of functional and conceptual actions were summed up 
(a)

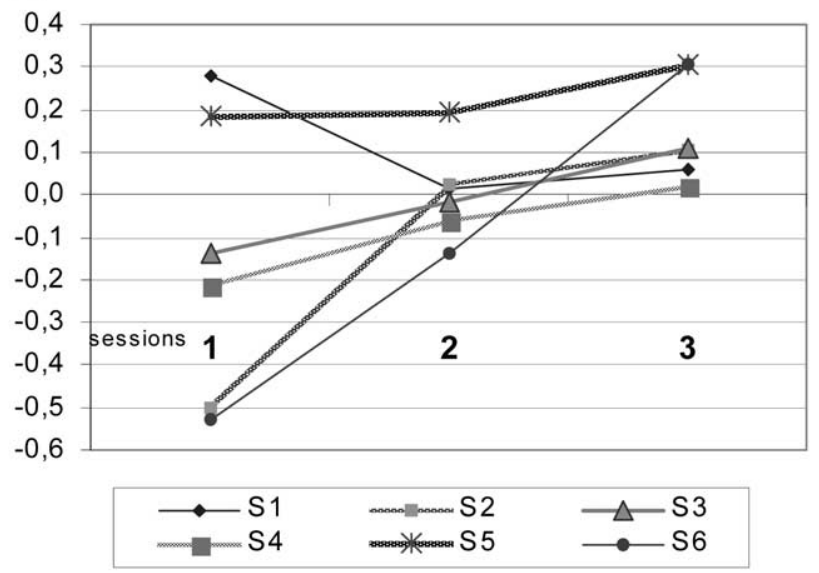

(b)

Figure 6 Normalized values

for perceptual actions related to (a) 'relations'( $b$ ) 'features' subcategory through three sessions for six subjects

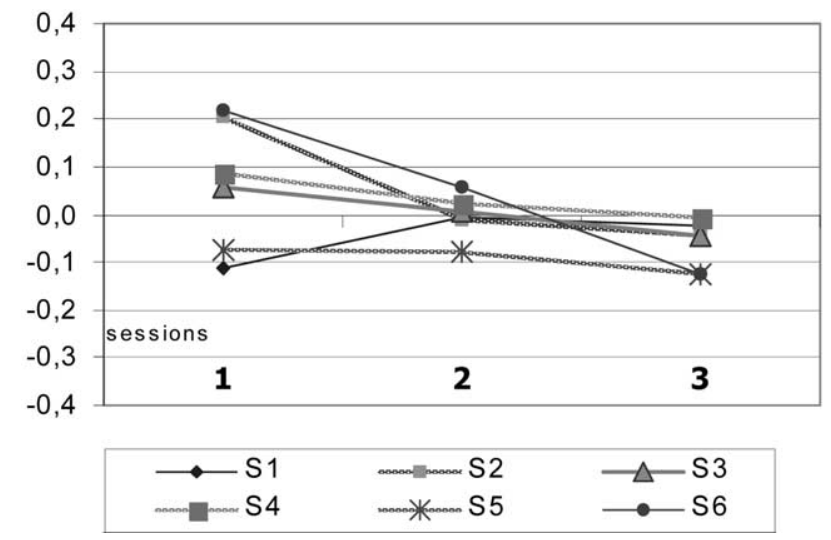

together in one chart (Fig. 7). It was observed that there was not a single tendency of designers' functional-conceptual actions in media transitions.

\section{Discussion}

\subsection{Results related to segmentation categories}

The first problem session had the highest number of segments due to the fact that the designer not only tries to understand what is required, but also chooses among various alternatives to obtain the best solution. When the second problem is introduced, it was expected that the designer will be more stable in his/her decision makings as s/he already knows what was expected. This results in a decrease in the number of segments because the intentions or goals would have fewer shifts compared to the first session. In 
(a)
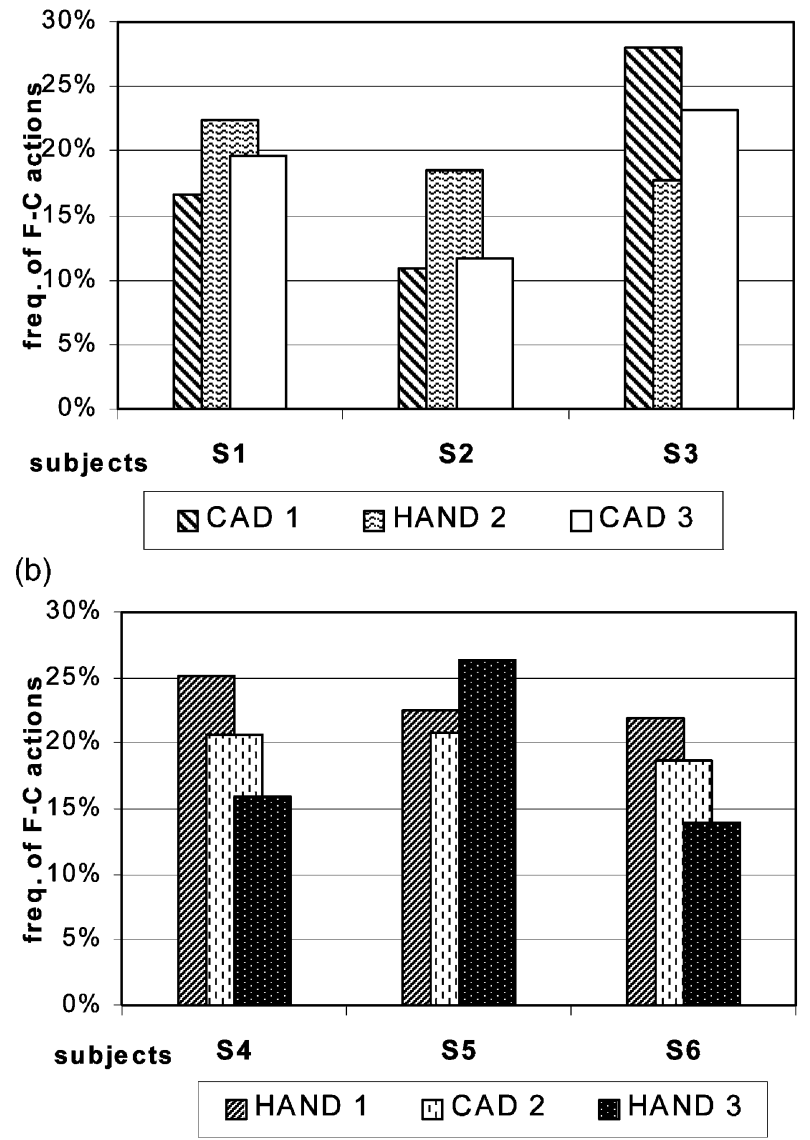

Figure 7 Frequency of func-

tional-conceptual $\quad(F-C)$

actions (a) CAD-HAND-

$C A D$ sessions (b) HAND-

CAD-HAND sessions

the third session, the number of segments is expected to decrease even more since it is the designer's second opportunity to work on the same problem. S/he already knew both what was expected and the outcome related to each. In this session, the designer might choose to work on a totally different alternative or on the same one by revising a number of parts. These preferences affected the number of segments in the third sessions.

The expected pattern of problem solving behavior can be seen in the HAND-CAD-HAND sessions (Fig. 2b), whereas CAD-HAND-CAD sessions depict a different pattern (Fig. 2a). Regarding to the increase in total number of segments in transition from CAD to HAND and the decrease in transition from HAND to CAD, traditional media has higher number of segments. Considering the ambiguous nature of hand sketches, this means designers changed their decision-makings and goals more frequently when they used the traditional media. 
It was observed that designers have a general tendency in solving the space-planning problem as simplifying it into two tasks. As the public area was indicated as entry (in the layout), the designer started to make a decision on the division of the layout plan for private and semi-private areas. Thus, the first thing to deal with was the global relations which determine private and semi-private areas regarding their spatial relations and the scenery. The second issue was the assessment of local relations among the space components found in private and semi-private areas.

Each designer was observed to have a different strategy in dealing with the assigned areas (public, private and semi-private). The period (in terms of segments) they spent to focus on certain areas changed. While dealing with the design of a certain area or component, some chose to shift to another part of the layout, and then return to that area again later on, while some of the designers finished the design of that area completely then started the design of the new area. During the design sessions, designers were observed to focus on an area in terms of segment numbers as:

- six or more segments

- two to five segments

- less than two segments.

The frequency of shifts in strategies depends on the designer's problem solving characteristics. There is not a single model to describe the frequency of shifts in the design process. Analyzing the subgoals in the strategy clusters, one could identify a profile of the problem-solving process. In the first two or three segments of the first problem sessions that can be called as the 'problem definition' state, designers deal with global relations and with a rough sketch of the local relations either with mental imagery or by external representations. This stage is usually very short in CADsketches, as the designer decides on a spatial relation model only in his/her mind, but longer in hand-sketches as the designer had the opportunity to have a diagrammatic representation of the spatial relations. Deciding on an area to deal with, designers most commonly define space components and spatial relations (A1, A2, D2-see Table 1), and redefine the space components (C1) or add elements to the space components (B1). When the designers revisit an area afterwards, they put things into the space components and organize them (A3, B1), or change the organization of the things inside $(\mathrm{C} 2)$ or redefine the space (C3). Sometimes E2 was observed in 'dealing with an area for the first time' category. Those sessions (that included E2) had a higher number of segments, because redefinition of spatial relations usually triggered other decisions, making the shifts in goals more frequent and this results in a higher number of segments. 
While analyzing the changes in the strategies of the designers, it was observed that F segment (copy the design) was usually followed by a redefinitive segment type (E or $\mathrm{C}$ type) in the hand sketch design sessions. The frequency of this case was $72 \%$ while the remaining implies the case that F was followed by other types of segments. So in many cases, over tracing the design seems to trigger redefinition of spaces or relations. In other words, designers tend to reinterpret some part of his/her sketch after the copying activity. Then redrawing the sketches might be acting as a tool to trigger visual thinking.

\subsection{Results related to Cognitive Actions (CA)}

The subjects' CA patterns (Fig. 3) are similar to the total number of segments patterns (Fig. 2). HAND-CAD-HAND sessions had a common decreasing pattern (Fig. 3(b)), which means a decrease in number of CA, possibly caused by the 'learning effect'. In Fig. 3(a) there is a CA increase in transition from CAD to HAND which demonstrates that designers' CA are also higher in traditional media.

Actually hand sketches include more physical actions (see Fig. 4), since the designer continuously draws and modifies and frequently copies the drawings to another sheet of paper. Whereas a lower frequency of the physical actions in hand sketching means that the other action categories (perceptual, functional-conceptual) have higher frequencies. Regarding these implications the reasons for higher frequency of the physical actions in CAD media for the first problem sessions is further discussed.

The increasing pattern in Fig. 6(a) shows that designers performed actions offered by the 'relations' subcategory in the proceeding stages of the design process which means, as far as they got more acquainted with the design problem, they started to associate more spatial and organizational relations between objects/elements. The decreasing pattern in Fig. 6(b) implies that designers tend to focus much more on the visual attributes of depictions and the spatial arrangement of the space components when they are not acquainted with the problem. The learning effect seems to decrease the focus on the visual and spatial features of depictions. In both subcategories of 'features' and 'relations', a major difference could not be observed, due to the change in design media.

\subsection{Variances among participants}

The richness of the verbal protocol was a varying component for each participant. This directly affected the encoding of segments and CA, as it was observed for Subject 4 (see Fig. 2(b) and 3(b)). In order to eliminate the variances in encoding depending on each participant's protocols, the 
pattern of the distributions was evaluated and the frequency (in percentiles) of the actions was analyzed.

Subject 2 displayed quite a high number of segments and CA (see Figs. 2 and 3). He considered himself to be more familiar and creative in traditional media, thus he produced many sketch drawings related to parts of the entire plan. So trying more alternatives while searching for a different form in designing, increased the number of shifts of his goals and the number of CA involved, compared to the others in the same group. Subject 3 tried some alternative forms also, and this can again be inspected in Fig. 2(a).

\subsection{Implications about the use of digital media}

The 3D view option in CAD environment enabled the designer to check the attributes, features, and organizational relations, while searching the alternatives to the questions like 'how the study desk looks near window', 'how that king size bed looks in that room' or 'how that antique basin looks in between the doors' ${ }^{26}$ (p. 69). So the participants had to make many evaluations conceptually related to the rich graphical imagery they are exposed to (in six of the nine CAD sessions). This was one of the factors which made the digital media time consuming, because designers had to deal with those attributes during conceptual design phase.

Although the designer was exposed to the various imagery characteristics of the digital media, s/he did not frequently tend to use most of them when $\mathrm{s} /$ he was actively sketching. One reason might be that the designers do not need a medium with excessive graphical images and features as they are adaptable to 'interactive imagery through sketching' which proposes 'systematic exchanges between conceptual and figural arguments' ${ }^{8}$ (p. 158) thus the representation medium has to be plain and ambigious so that interactive imagery could become possible during conceptual design. Secondly those designers, during their education, had been acquainted with working on plan drawings and experiencing most of the 3D features in their mental imagery as one of the participants stated: "I always see the plan in 3D, as if the walls are elevating; when I work on the plan I see also the perspective views"26 (p. 69). So some features of the software might remain unused due to their habitual activities in the traditional media. This also brings the evidence that the subjects were still novice users of CAD although they had the software training programme.

26 Bilda, Z 'Designers' cognition in traditional versus digital media during conceptual design' Unpublished MFA Thesis, Bilkent University, Ankara (2001).
Similar to the total number of segment categories, the total number of cognitive actions was also relatively higher in traditional media. This result can be explained by designers' mode of thinking and reasoning in different 
media. Firstly, designers have always used hand sketches as a cognitive tool throughout their education, which in turn might limit their cognitive interaction with the digital media. Secondly, the commercial CAD software is inflexible to support designers' habitual activities such as doodling, drawing diagrams, gesture of moving pencil, copying activity and so on which all seem to be evidences of visual thinking and reasoning.

\subsection{Implications about the 'learning effect'}

In the experiment, the first and the third design sessions employed the same design problem, thus the third session was a second opportunity of the designer to work on the same problem. The decrease in numbers of shifts in goals/intentions (segmentation categories) and of the CA was observed in transition from first problem session to the third one. The decrease in both cases was explained by designers' learning, named the 'learning effect'. The negative slope of the learning effect was observed to be smaller in HAND-CAD-HAND sessions but higher in hand-cad-hand sessions. This situation implies that the media affected the learning. If the participants were designing in a media that they were more acquainted with (that is traditional media), then 'learning effect' seems to be amplified.

\subsection{Comparison with related work}

The earliest form of the coding scheme used in this study was devised by Suwa and Tversky ${ }^{13}$. The revised coding scheme by Suwa et al. ${ }^{14}$ was a more detailed taxonomy, constituting of indexes to systematically inspect the links between the cognitive actions. However, the study by Suwa et al. ${ }^{14}$ did not analyze the internal links, but explored the correlations between different types of actions.

The present study did not employ any of the analyses mentioned above due to the media transition that has to be considered separately. As the major concern of the study is the comparison of media, it was reasonable to utilize the general taxonomy by Suwa et al. ${ }^{14}$ by making revisions on the coding scheme to fit to a comparative analysis. At the same time, the design process in the empirical research was to be closer to a problem solving process in order to easily observe the contents of the designers' actions. Introducing an interior space-planning problem with specifications makes the inspection of design sessions more systematic, as the entire problem solving process could be decomposed into goals (in terms of designers' intentions). In this study the definition of segment was related to these goals/intentions of designers, whereas in the studies by Suwa and Tversky ${ }^{13}$ and Suwa et al. ${ }^{14}$ each design move (which is the smallest component) was defined to be a segment. As the present study aimed at analyzing six participants' design protocols each having three consecutive 
design sessions in different media, the segmentation and encoding of cognitive actions were handled in optimum detail level. So a segment in this study might be composed of a number of 'design moves', and further the start-end of a new segment is identified depending on the segmentation categories scheme (Table 1). Although the typical segments in the present study seem much bigger to analyze the cognitive features of thought they allow the interpretation of the design behavior in terms of goals and problem solving strategies.

\section{Conclusion}

This study was an attempt to model and analyze the effects of media on designers' sketching, where their design thoughts during the solution of an interior space-planning problem were examined by the method of retrospective protocol analysis. Another goal of the study was to decompose the entire problem solving process into typical segments depending on designers' intentions. The vocabulary needed to identify the designers' primitive actions was reassessed from Suwa et al.'s ${ }^{14}$ coding scheme. The analysis of segmentation and cognitive action categories enabled an insight on design thinking and making in traditional versus digital media.

The segmentation categories scheme devised was independent of the design sessions of different media, which means it acted as a general taxonomy that helped to model the specific problem solving process. Using the segmentation categories scheme, the problem solving strategies of designers were analyzed and it was observed that there was no tendency to use specific strategies for all participants throughout the design sessions. Rather it was observed that all participants had some common subgoals while dealing with specific tasks. Analyzing the sequence of subgoals in the strategy clusters, a significant result depicted that 'copying the design' activity was usually followed by redefining spaces or relations, which is a signal of re-interpretation. Strategy clusters also revealed that designers mostly associated global spatial relations effectively when they work with freehand sketches, as the digital media was not flexible to allow a doodling activity or a diagrammatic representation. These two conclusions provide implications for the structure of conceptual CAD packages for architects that the system should offer a semi-transparent copying activity like a tracing paper provides, and a tool for diagrammatic representation.

Designers' sketching activity seemed to have different dynamics in different representational media in terms of design thinking and making. The first significant difference was that designers' goals and intentions more frequently changed in traditional media (Fig. 2). This situation was sup-

ported by Goel's ${ }^{11}$ findings and explained as the ambiguous nature of 
sketches and the designers' habit of using sketches as a representational media throughout their education.

Similar to the total number of segment categories, the total number of cognitive actions was also relatively higher in traditional media. This result cannot be interpreted as a tendency to think, see, perceive less in digital media, but as designers varying mode of thinking and reasoning in different media. Regarding that designers have always used hand sketches as a cognitive tool to interact with throughout their education, this might be limiting their cognitive interaction with the digital media. Further the current software was inadequate to support their habitual sketching activities.

The current commercial CAD software usually works on 'draw and then modify' principle, which was observed in designers' physical actions, such that 'modify' actions were more frequently used in digital media. The use of 3D interactive visualization feature in digital media did not significantly affect the evaluation and implementation of functional criteria in the specific design problem.

It can be concluded that designers were more effective in using time, conceiving the problem, producing alternative solutions and in perceiving the visual - spatial features and the organizational relations of a design in traditional media rather than digital media during conceptual design. Although digital media seems to be inconvenient for the conceptual design phase, this situation depends on designers' designing habits and the inflexibility of the CAD software.

As concluded previously, conventional CAD software does not yet support the early conceptual design phase but mental imagery could be a tool for that. Then a design medium could be defined as an environment where the designer creates a virtual model in his/her mind and then makes the simulation digitally. As a further study, the interaction between mental imagery and 3D CAD environment could be analyzed as both tools aim to work with a virtual model; while mental imagery creates, the latter externalizes the output. Analyzing this kind of a design medium might propose new forms of interaction between mental imaging and simulation in digital media. Re-inventing the nature of cognitive processes involved in such a medium might support development of conceptual computer-aided architectural design packages, as well as proposing a new methodology for design education. 


\section{Acknowledgements}

The authors would like to thank Prof. John Gero for his valuable guidance to the idea of this research on design cognition and for his insightful comments on the design of the experiment. Special thanks to Prof. Gabriela Goldschmidt for being so kind to review and criticise Bilda's MFA thesis, so that we have repeated the experiment for one subject and reassessed the analysis of the all protocols. Thanks to Dr. Manolya Kavakli for sharing her experience with us in the process of analysing the protocols. 